\title{
Cause of dysphagia in elderly with dementia: pancreatic tail neoplasm
}

\author{
Patricia López Pardo* \\ Geriatric Medicine Department, Hospital Universitario Puerta de Hierro-Majadahonda, Majadahonda, Madrid, Spain
}

\begin{abstract}
It describes dysphagia secondary to metastatic involvement of the esophagogastric junction, in a clinical case with several poorly described relationships: dementianeoplasia of pancreatic tail-infiltration of the esophagogastric junction.
\end{abstract}

\section{Clinical images}

A 93-year-old woman referred from a nursing home by weight loss, fatigue, dysphagia for solids and liquids and sialorrhea after swallowing from 6 months ago. In recent weeks, epigastralgia associated with intakes. As medical history: left hip fracture 2 years ago, moderatestage dementia with recent memory impairment of more than 2 years of evolution. She walks short routes with walker, precise help for rest of basic activities of daily living. Physical examination, with no significant findings. No analytical alterations. Tumor markers, negative. Abdominal CT with intravenous contrast in arterial and portal venous phases: infiltrating mass compatible with primary pancreatic tail neoplasia (Figure A), with left paraaortic adenopathies and extensive gastric, esophagogastric junction (Figure B) and visceral perivascular infiltrations. During her stay the patient presents hypoactive delirium, inability oral feeding, vomiting of bilious and undigested food content, with a good response to haloperidol [1]. Moved to Palliative Care Unit for control and management of symptoms.

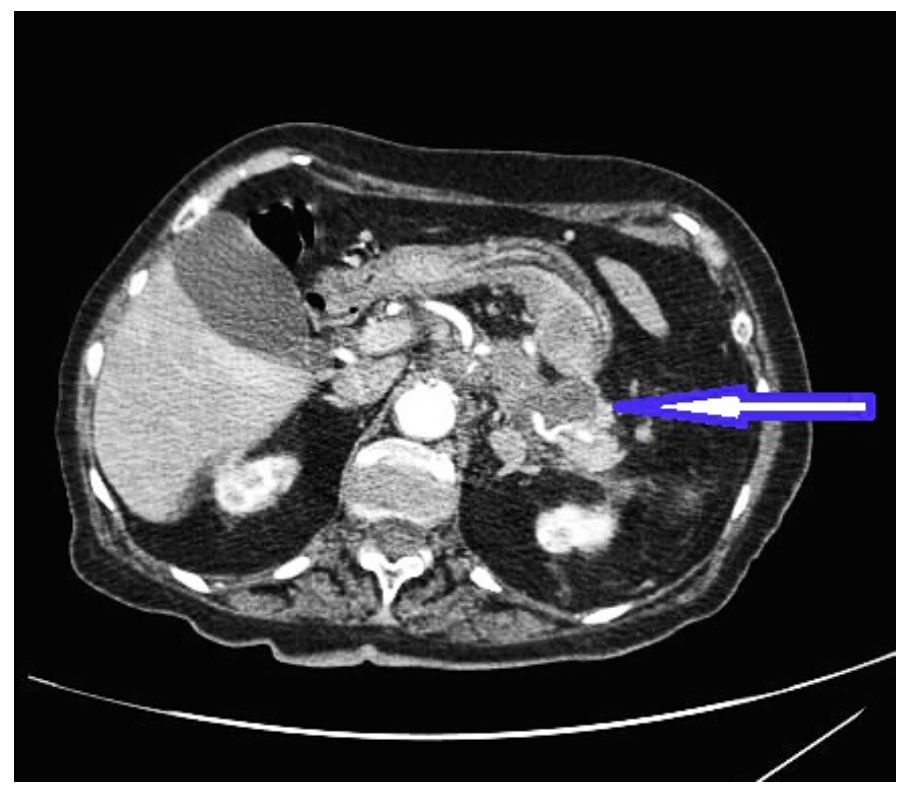

Figure A. infiltrating mass compatible with primary pancreatic tail neoplasia

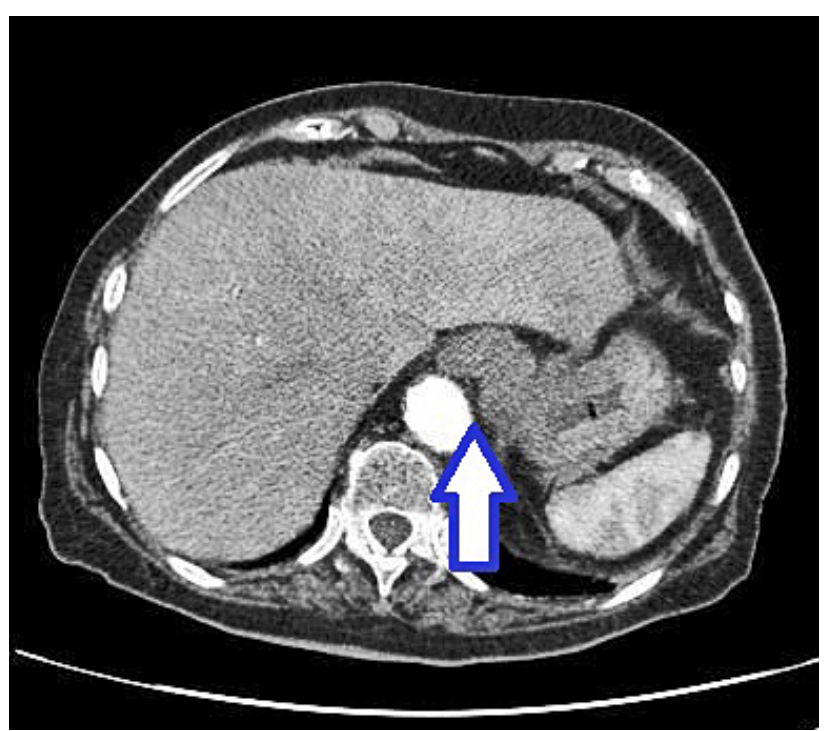

Figure B. Left paraaortic adenopathies and extensive gastric, esophagogastric junction

\section{Conclusion}

It is important to assume that, in patients with dementia who suffer from dysphagia, the etiology is not just neurogenic. In this case, it describes dysphagia secondary to metastatic involvement of the esophagogastric junction, in the inverse association between cancer and neurodegenerative disease [2].

\section{Conflicts of interest}

None declared

Correspondence to: Patricia López Pardo, Geriatric Medicine Department. Hospital Universitario Puerta de Hierro-Majadahonda, Majadahonda, Madrid, Spain; Email: plpardo@hotmail.com

Key words: pancreatic neoplasms, esophagogastric junction, dementia, aged, deglutition disorders

Received: August 20, 2017; Accepted: September 12, 2017; Published: September 15, 2017 
Pardo PL (2017) Cause of dysphagia in elderly with dementia: pancreatic tail neoplasm

\section{References}

1. Murray-Brown F, Dorman S (2015) Haloperidol for the treatment of nausea and vomiting in palliative care patients. Cochrane Database Syst Rev 11: CD006271.
2. Driver JA (2014) Inverse association between cancer and neurodegenerative disease: review of the epidemiologic and biological evidence. Biogerontology 15: 547-557. [Crossref]

Copyright: (C2017 Pardo PL. This is an open-access article distributed under the terms of the Creative Commons Attribution License, which permits unrestricted use, distribution, and reproduction in any medium, provided the original author and source are credited. 